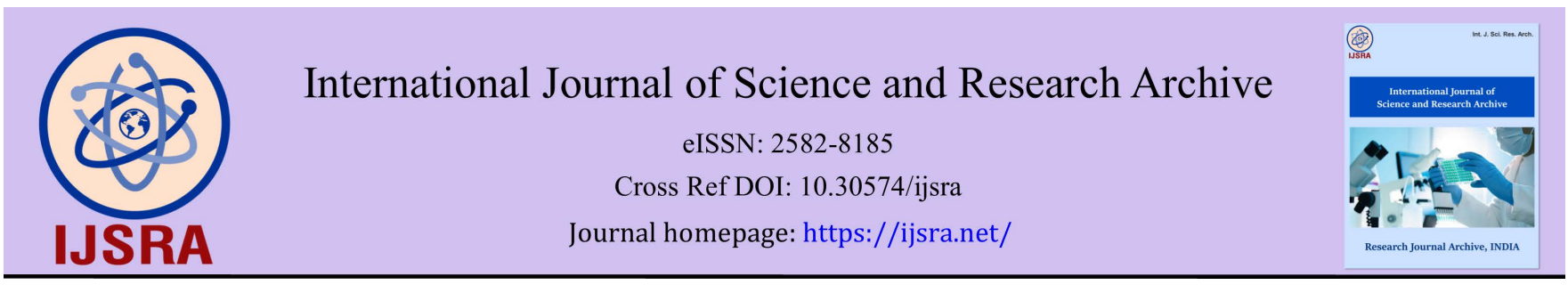

(CASE REPORT)

Check for updates

\title{
Rare case of radial artery pseudoaneurysm protruding through skin after a single arterial puncture for blood-gas analysis
}

\author{
Dimitrios Giotis 1, ${ }^{*}$, Vasileios Panagiotopoulos ${ }^{1}$, Christos Konstantinidis ${ }^{1}$, Paraskevi Vravoritou 1, Stefania \\ N. Karampina ${ }^{2}$, Vasiliki Telaki ${ }^{2}$, Christos Kotsias ${ }^{1}$, Varvara-Sylvana Kardakari ${ }^{1}$, Dimitrios Vardakas ${ }^{1}$ and \\ Sotiris Plakoutsis ${ }^{1}$
}

${ }^{1}$ Orthopaedic Department, General Hospital of Ioannina “G. Hatzikosta”, Ioannina, Greece.

${ }^{2}$ Cardiology Department, General Hospital of Ioannina "G. Hatzikosta", Ioannina, Greece.

International Journal of Science and Research Archive, 2021, 02(02), 105-109

Publication history: Received on 27 March 2021; revised on 30 April 2021; accepted on 03 May 2021

Article DOI: https://doi.org/10.30574/ijsra.2021.2.2.0059

\begin{abstract}
The pseudoaneurysm of the radial artery is an infrequent complication mainly after iatrogenic procedures, with an increased incidence over the last years. The aim of the current study was to present a rare case of a radial artery pseudoaneurysm that projected through the skin after a single arterial puncture for blood-gas analysis. A 79-year-old man, with a history of atrial fibrillation and coronary heart disease on anticoagulants, was admitted to the cardiological intermediate care unit, with pulmonary oedema due to heart failure deregulation. On the next day, his left radial artery was punctured for blood-gas analysis. No other catheterization of the vessel was executed in the following days. Six days later a pulsatile, palpable mass on the skin surface, over the site of puncture, was recognized. Subsequently a Doppler ultrasound that was performed, confirmed the diagnosis of pseudoaneurysm. The pseudoaneurysm was surgically removed under general anesthesia. The patient was hospitalized for four days for trauma monitoring and intravenous antibiotics and afterwards he was discharged from the Hospital. Three months postoperatively, no sign of recurrence was observed. The pseudoaneurysm of the radial artery is an uncommon complication that might occur even after simple iatrogenic procedures, like after a direct arterial puncture for blood-gas analysis. Therefore, specialists should always be aware of this complication in similar cases.
\end{abstract}

Keywords: Pseudoaneurysm; Radial artery; Blood-gas analysis; Complication; Iatrogenic procedures.

\section{Introduction}

The pseudoaneurysm of the radial artery is a rare complication after trauma or invasive iatrogenic procedures, such as the transradial catheterization in coronary angiographies which could cause arterial wall lesions [1-3]. This transradial approach has become more preferable than the transfemoral approach over the last years, due to its safer profile and fewer complications, which allow nearly immediate patient mobilization after the procedure [1, 4, 5]. Risk factors related to pseudoaneurysm occurrence during catheterization of the radial artery include older age, coagulation disorders, use of antiplatelet/anticoagulant drugs and long duration of the technique.

Disruption of the arterial wall might be caused by even a single arterial puncture. To the best of our knowledge, there are only few cases that report formation of pseudoaneurysm after a single arterial puncture for blood-gas analysis [67]. The purpose of the present study was to present an uncommon case of a radial artery pseudoaneurysm projecting through the skin, after a single direct arterial puncture, which was treated operatively.

\footnotetext{
* Corresponding author: Dimitrios Giotis

Orthopaedic Department, General Hospital of Ioannina "G. Hatzikosta”, Ioannina, Greece.
}

Copyright (C) 2021 Author(s) retain the copyright of this article. This article is published under the terms of the Creative Commons Attribution Liscense 4.0. 


\section{Material and methods}

A 79 year-old male, with a history of ischemic heart failure, atrial fibrillation and coronary heart disease on anticoagulants presented to the Emergency Department after sustaining acute pulmonary oedema due to heart failure deregulation and was admitted to the cardiological intermediate care unit. On the second day of hospitalization, his left radial artery was punctured for arterial-blood-gas analysis as a routine workup. A 23-gauge needle was used and only one puncture was attempted to collect the sample. Compression of about 3-4 minutes with bandage was applied over the punctured site. No other catheterization of the vessel preceded or followed.

On the next day, the patient was transferred to the ward in the Cardiology Department. Five days later he complained of pain and swelling over the radial volar puncture site. Clinically, a pulsatile, palpable, soft mass on the skin surface, over the site of puncture was revealed, without any inflammation sign (Figure 1). The pulse of the left radial artery was palpable proximally and distally of the formation. Allen's test demonstrated that ulnar artery alone was sufficient for the hand's blood perfusion. His hand movement was not restricted and no other complications such as neurapraxia, or compartment syndrome were found. On ultrasonography, the lesion appeared to be cystic in nature and nearly circular in shape displaying a maximum diameter of $1.5 \mathrm{~cm}$ with turbulent flow, concentric wall thrombus, functional lumen of $0.6 \mathrm{~cm}$, and thickness of the stem that fed it at $0.2 \mathrm{~cm}$ (Figure 2A/B). Thus, the Doppler ultrasound confirmed the diagnosis of pseudoaneurysm and an orthopedic consultation was requested.

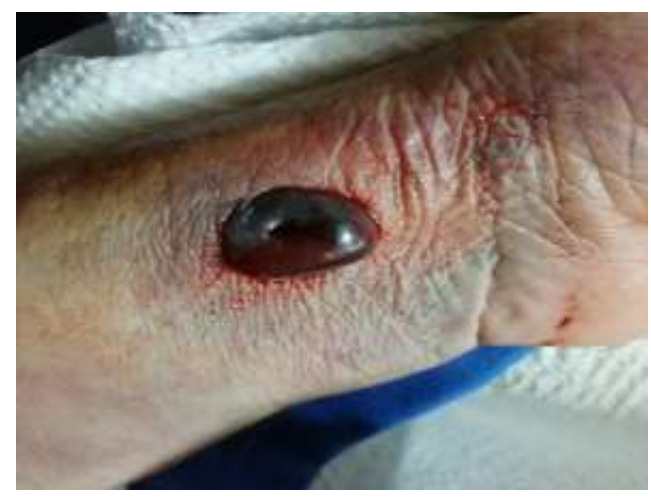

Figure 1 Pseudoaneurysm as depicted preoperatively

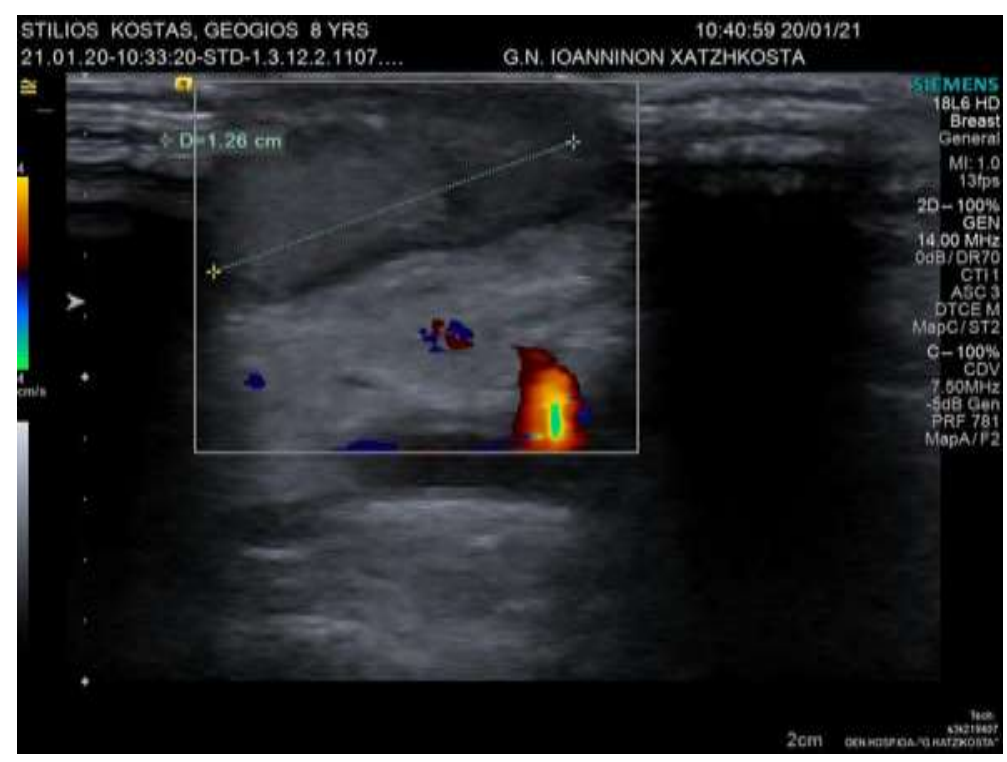

Figure 2A Ultrasound image of radial artery pseudoaneurysm 


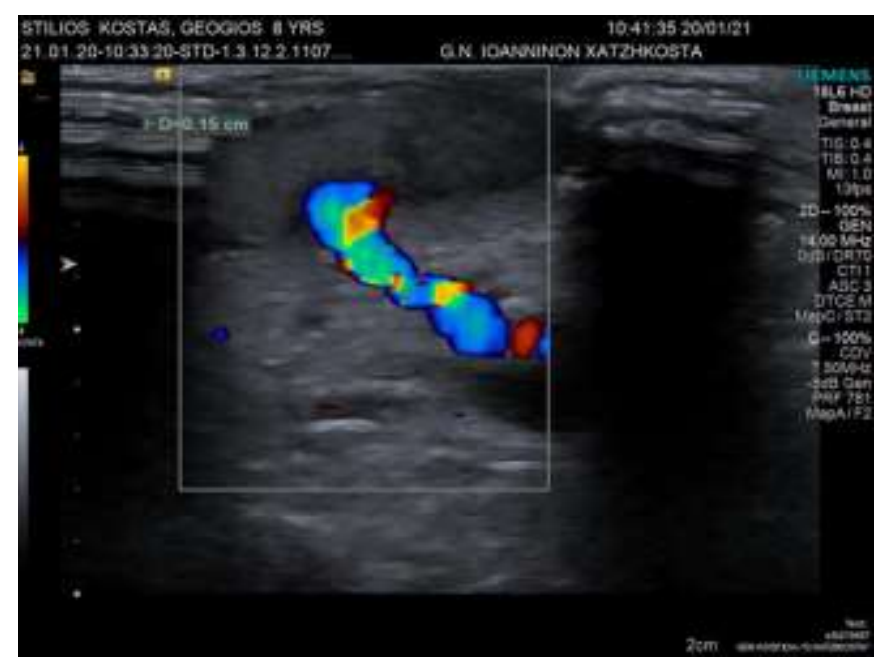

Figure 2B Ultrasound image of radial artery pseudoaneurysm

\section{Results and discussion}

Surgical treatment was performed three days later. Forty-eight hours prior to operation, anticoagulant therapy was interrupted. Intraoperatively, the left wrist exploration revealed a pseudoaneurysm with pinpoint bleeding at the site of arterial puncture in the left radial artery. The pseudoaneurysm was removed after intersecting the aneurysm pedicle and primary repair was performed at the site of hemorrhage (Figure 3). There were no postoperative complications and patient's post-surgical radial pulse was normal. He remained for four days at the Cardiology Department for trauma monitoring and intravenous antibiotics and afterwards he was discharged from the Hospital. Three months later at the scheduled follow-up, the patient did not show recurrence of the lesion or any further complication.

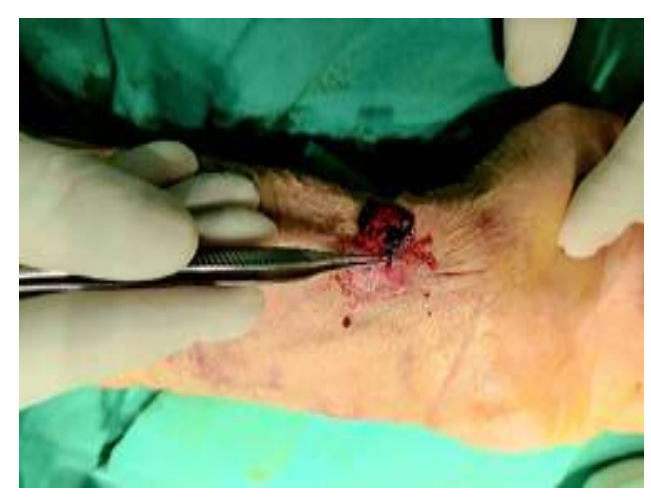

Figure 3 Intraoperative view of the cystic lesion

Pseudoaneurysm formation is an infrequent complication with a reported incidence of $0.2-3 \%$ after a transfemoral approach and less than 0.01 after a transradial catheterization [8]. Iatrogenic factors such as multiple puncture efforts, insufficient hemostasis, vascular site infection, systemic use of anticoagulants and elderly age may increase the risk of pseudoaneurysm development [8-10].

This lesion may occur after a rupture through all layers of the artery with hemorrhage and hematoma formation between the vessel wall and surrounding parenchyma. The cavity that is created remains in continuity with the normal artery as a false sac, which is lined with endothelium. Its outer walls are consisted of fibrous scar tissue [6]. However in the current scenario, pseudoaneurysm was the result of a single puncture of radial artery for arterial blood-gas analysis that protruded through the skin on the radial puncture site. To our knowledge, there are no similar cases reported in the literature. 
Leone et al. reported a case of radial artery pseudoaneurysm complicating a sole puncture for blood-gas analysis. They regarded that an incorrect compression of the vessel after the puncture was the main reason for this formation, which was treated with excision of palpable mass and suture of the defect of radial artery`s wall [6]. Similarly, Patel et al. also reported the development of a pseudoaneurysm after a single puncture for blood-gas analysis that was treated conservatively with close follow-up due to its small size and asymptomatic nature [7].

Clinical signs and symptoms for the diagnosis of a radial artery pseudoaneurysm are that of an often painful, palpable mass commonly but not always, and pulsatile. In our case, clinical examination revealed palpable pulse both proximally and distally to the cystic lesion. Concerning the imaging diagnostic tools, Doppler ultrasound which is an inexpensive, fast and effective imaging method, is the most commonly used diagnostic technique for the detection of these cystic lesions [6-10]. In cases of difficulties, magnetic resonance imaging (MRI), three-dimensional computed tomography (CT) angiography or gandolinium-enhanced magnetic resonance angiography will set the diagnosis by offering visualization of the mass from all angles and in any projection [7-10]. In our patient, the diagnosis was established with the use of ultrasonography which indicated the presence of pulsatile, turbulent flow along with a hematoma with variable echogenicity, pathognomonic signs for pseudoaneurysm.

Regarding the management of radial artery pseudoaneurysm, there have been proposed many therapeutic options which vary according to the size, location or any other anatomic feature of the lesion, the etiology, the blood flow status proximally and distally to the lesion and the presence of any other associated symptoms [11]. Nonsurgical treatment, that requires thrombus formation, includes clinical observation, mechanical compression with bandages, ultrasoundguided compression repair, ultrasound-guided percutaneous thrombin injection or endovascular therapy [5, 11-12].

Generally, in cases of lesions, relative small in size $(<1 \mathrm{~cm})$ which have been detected early, conservative treatment can offer a reliable outcome as the majority of them will be thrombosed within 3-4 weeks [5, 11-12]. On the contrary, surgical intervention is needed in cases of large lesions $(>2-3 \mathrm{~cm})$, in complicated cases or when nonsurgical treatment fails. In our case, the relative large size of the cystic formation, in association with the mass protruding through the skin, led to the decision of surgical management.

\section{Conclusion}

Pseudoaneurysm of the radial artery is considered as an infrequent complication that might occur not only after catheterization of radial artery but also after basic iatrogenic procedures such as a single direct arterial puncture for blood-gas analysis. Therefore special attention is demanded by clinicians when they perform even simple medical procedures in order to avoid similar iatrogenic complications.

\section{Compliance with ethical standards}

\section{Disclosure of conflict of interest}

All authors declare that they have no competing interests.

\section{Statement of informed consent}

The patient gave his informed consent for participation in accordance with our Institutional Review Board procedures.

\section{References}

[1] Kiemeneij F, Laarman GJ, Odekerken D, Slagboom T, Van Der Wieken R. A randomized comparison of percutaneous transluminal coronary angioplasty by the radial, brachial and femoral approaches: the access study. J Am Coll Cardiol. 1997 May; 29(6): 1269-75.

[2] Poonai N, Lim R, Lynch T. Pseudoaneurysm formation following a traumatic wrist laceration. CJEM. 2011; 13: 4852.

[3] Ranganath A, Hanumanthaiah D. Radial artery pseudo aneurysm after percutaneous cannulation using Seldinger technique. Indian J Anaesth. 2011; 55: 274-6.

[4] Blanco A, Shayesteh S, Chu LCH, Fishman EK. Radial artery pseudoaneurysm after transradial cardiac catheterization: A case presentation. Radiol Case Rep. 2020 Sep 7; 15(11): 2213-2216. 
[5] Zegrí I, García-Touchard A, Cuenca S, Oteo JF, Fernández-Díaz JA, Goicolea J. Radial artery pseudoaneurysm following cardiac catheterization: clinical features and nonsurgical treatment results. Rev Esp Cardiol (Engl Ed). 2015; 68(04): 349-351.

[6] Leone V, Misuri D, Console N. Radial artery pseudoaneurysm after a single arterial puncture for blood-gas analysis: a case report. Cases J. 2009 Jul 21; 2: 68-90.

[7] Patel KN, Gandhi SP, Sutariya HC. Radial artery pseudoaneurysm: A rare complication after a single arterial puncture for blood-gas analysis. Indian J Crit Care Med. 2016 Oct; 20(10): 622-626.

[8] Zankl AR, Andrassy M, Volz C, Ivandic B, Krumsdorf U, Katus HA, Blessing E. Radial artery thrombosis following transradial coronary angiography: incidence and rationale for treatment of symptomatic patients with lowmolecular-weight heparins. Clin Res Cardiol. 2010 Dec; 99(12): 841-7.

[9] Kanei Y, Kwan T, Nakra NC, Liou M, Huang Y, Vales LL, Fox JT, Chen JP, Saito S. Transradial cardiac catheterization: a review of access site complications. Catheter Cardiovasc Interv. 2011 Nov 15; 78(6): 840-6.

[10] Collins N, Wainstein R, Ward M, Bhagwandeen R, Dzavik V. Pseudoaneurysm after transradial cardiac catheterization: case series and review of the literature. Catheter Cardiovasc Interv. 2012; 80: 283-287.

[11] Kiat JTC, Lung AWS, Tang CC. When one complication begets another-A case report of radial artery pseudoaneurysm following radial artery occlusion post-transradial coronary angiography. J Cardiol Cases. 2020 Apr 25; 22(2): 52-54.

[12] Kongunattan V, Ganesh N. Radial artery pseudoaneurysm following cardiac catheterization: A nonsurgical conservative management approach. Heart Views. 2018; 19(2): 67-70. 